\title{
STANDARDIZATION OF CHIMNEY WEIR FOR REGULATING WASTE WATER IN GRIT CHAMBERS
}

\author{
C.Rangaraj $^{1}$, M.Raghunath ${ }^{2}$, L Sampath Kumar ${ }^{3}$ \\ ${ }^{1}$ Sri Siddhartha Institute of Technology, Tumakuru, 572105, India \\ ${ }^{2}$ Government Engineering College, K R Pete, , India \\ ${ }^{3}$ Sri Siddhartha Institute of Technology, Tumakuru, 572105, India
}

\begin{abstract}
It was aimed to lend confidence to prospective users of Weirs pioneered in Indian Institute of Science Bangalore by Standardization which consists of testing the flow measuring instrument under varying conditions, so that range of application could be trusted eliminating any unpleasant surprises highlighted which could be awaiting the user working beyond the recommended range. The experimental work in I.I.Sc was restricted to just a few weirs. Chimney weirs find applications in grit chamber outlets since they maintain a constant average velocity of flow whatever may be the depth of flow. This unique property of constant average velocity allows uniform settlement of grit whatever may be the depth of flow. But for the industry to use the weir the work of standardization has to be undertaken.
\end{abstract}

The work of standardization includes the determination of sensitivity of the experimental parameters (here coefficient of discharge $C d$ ). This work was undertaken in the new channel funded by VTU. Eccentricities were imparted to the weir both in horizontal and vertical direction and sensitivity of $C d$ and variation of $C d$ with these eccentricities were experimentally determined. Total numbers of one plus ten weirs were fabricated for the determination of cd for two batches of students. Measured discharge.

Keywords: Standardization, Chimney, Weir

\section{INTRODUCTION}

Thin plate weirs or sharp crested weirs are the most frequently used flow measuring device as they most accurately measure the discharge as compared to other discharge measuring instrumentation. In addition they are inexpensive. Chimney weir profile consists of a rectangular weir or vertical slot over an inward trapezium. The Chminey weir gives a linear discharge within a permissible band of error. The task was to fix the shape of the weir by optimization such that the range of head over which the head-dicharge relationship is linear within the prefixed error. Additionally it is not permitted that in its linearity range nowhere the error band is violated. A new and more elegant optimization procedure developed in IISc named "Geometrical Oscilating point method" is used to analyze the discharge-head relationship in the weir. It is shown that a rectangular weir placed over an inverted V- notch of depth $0.90 \mathrm{~d} 1$ gives maximum operating range. For all flow in the rectangular portion, the discharge is proportional to the linear power of the head measured above a reference plane located at $0.29 \mathrm{~d} 1$ below the weir crest; in the range of head, $0.90 \mathrm{~d} 1<\mathrm{h}<7.47 \mathrm{~d} 1$, within a maximum error of $\pm 1.5 \%$ from the theoretical discharge. The optimum range of operation of the newly designed weir is $200 \%$ greater than in the chimney weir designed by Keshava Murthy and Giridhar 1990); and is nearly 950\% greater than in the inverted V-notch. Experiments with two weirs having half crest widths of $0.10 \mathrm{~m}$ and $0.12 \mathrm{~m}$ had confirmed the theory by giving a constant average coefficient of discharge of 0.634. The application of the weir in the design of rectangular grit chamber outlet is 117 aximized 117 in that the datum for the linear discharge-head relationship is below the crest level of the weir. The effect of variation of the/width of the rectangular weir on $\lambda$ is studied, in order to 117 aximize it; since it is desirable to have higher values of $\lambda$ to 117 aximize the space for the collection of grit.

$V_{\text {ave }} \alpha \frac{\mathrm{h}+\lambda \mathrm{d} 1}{\mathrm{WgHg}}$

where $\lambda \mathrm{d} 1$ is the datum from which the heads are measured in the weir, and hg and $\mathrm{Wg}$ are the depth of flow and width of the rectangular channel. The significance of the datum in the design of weirs was demonstrated by Keshava Murthy and Seshagiri (1968). For the velocity to be constant for all depths of flow $\mathrm{Hg}$ should be equal to the $\mathrm{h}+\lambda \mathrm{d} 1$.

The Sutro weir is currently in use in rectangular grit chambers. Since the weir has to be fixed at the bed of the channel, to maintain a constant average velocity in the grit chamber, there is no gap between the crest of the weir and the bed of the channel and sediment may get washed over. This drawback was overcome by Keshava Murthy and Seshagiri [1968] by the design of the orifice notch. In the present weir (a geometrically simple weir) it is achieved by fixing the weir crest in such a way that the reference plane of the weir coincides with the bed of the crest. This is possible because the reference plane of measurement of head lies below the crest. The space between the crest of the weir and the bottom of the channel allows the collection of grit. 


\section{FORMULATION}

The discharge through the chimney weir, neglecting the velocity of approach and surface tension effects, is given by (Keshava Murthy and Giridhar 1990).

$\mathrm{Q}(\mathrm{h})=2 / 3 \mathrm{C}_{\mathrm{d}} \sqrt{ } 2 \mathrm{~g} 2 \mathrm{~W}_{\mathrm{c}} \mathrm{h}^{3 / 2}-8 / 15 \mathrm{C}_{\mathrm{d}} 2 \mathrm{gtan}(\theta) \mathrm{h}^{5 / 2}$

$\mathbf{0} \leq h \leq p$

$q(h)=2 / 3 C_{\mathrm{d}} \sqrt{ } 2 \mathrm{~g} 2 \mathrm{~W}_{c} h^{3 / 2}-8 / 15 \mathrm{C}_{\mathrm{d}} 2 \mathrm{gtan}(\theta)\left[h^{5 / 2}-\right.$ $\left.(h-p)^{5 / 2}\right) ; p \leq h \leq \mathrm{h}_{\max }$

When ' $h$ ' is not too small; where, lh' = head above the weir crest; 'Wc = half crest width; 'p' = height of the trapezium; $=$ side slope of the trapezium (half vertex angle of the corresponding Inverted V-notch (IVN)); ' $\mathrm{g}=$ acceleration due to gravity; and $\mathrm{Cd}=$ Coefficient of discharge.

The coefficient of discharge is assumed to be constant, which is generally true for sharp crested weirs and streamlined flows. The coefficient is a function of several parameters, including the head causing flow, dimensions of the weir in relation to the dimensions of the channel and the crest height. The value of $\mathrm{Cd}$ is ascertained from experiments as in conventional weirs and the variation of it will generally be within $\pm 1 \%$ of the average, when $h$ is not too small. The exact value of $\mathrm{Cd}$ has to be ascertained by detailed experiments which are necessary for the 118aximized118ation of the weir, which is outside the scope of this thesis.

Eqs. 2 and 3 can be expressed for convenience in the nondimensional form as

$\mathrm{Q}(\mathrm{H})=2 / 3 \mathrm{H}^{3} /^{2}-4 / 15 \mathrm{H}^{5} r^{2} ; \quad 0 \leq H \leq P$

$Q(H)=2 / 3 H^{3 / 2}-4 / 15(H-P)^{5 / 2} ; \quad H \geq P$

where, $Q=q /\left(2 C_{d} \sqrt{ } 2 g d_{l}^{5 / 2} \tan (\theta)\right) ; H=h / d_{l}$ and $\mathrm{P}=\mathrm{p} / \mathrm{d}_{1}$.

The graph Q vs. H was plotted in for various $\mathrm{P}$. It is seen to be nearly linear over a wide range of head, particularly for $\mathrm{P}$ $=0.9$. Hence it is possible to obtain a discharge-head relationship of the form [within the bounds of error]

$Q_{L}=m H+C$

where, $\mathrm{m}$ and $\mathrm{C}$ are the slope and intercept of the straight line in the Q vs. H plot. It is ensured that the relative error ' $\mathrm{e}$ ' does not exceed a prefixed maximum percentage error emax ' in its range of operation, i.e.,

$e=Q Q-Q n / / Q^{x} 100 \leq \mathrm{e}_{\max }$

In most discharge measurements in practice involving weirs and notches, emax $=2$ is normally allowed (Troskolanski 1960). This results in a reasonably high degree of accuracy under field conditions. Hence emax $=1.5 \%$ adopted in is quite reasonable.

\section{LINEAR FITTING}

Eqn.7 is used to define a permissible region within which the straight line (Eqn.6) is positioned. It follows that QL has to lie strictly within the permissible region bounded by

$\mathrm{f}_{1}(\mathrm{H})=\mathrm{Q}(\mathrm{H})\left(1+\mathrm{e}_{\max / 100}\right)$

$\mathrm{f}_{2}(\mathrm{H})=\mathrm{Q}(\mathrm{H})\left(1-\mathrm{e}_{\max / 100}\right)$

In its range of operation [linearity range.LR). In the case of linear weirs $\mathrm{f} 1(\mathrm{H}), \mathrm{f} 2(\mathrm{H}) \& \mathrm{Q}(\mathrm{H})$ correspond to $\mathrm{y} 1(\mathrm{x}), \mathrm{y} 2(\mathrm{x}) \& \mathrm{y}(\mathrm{x})$ respectively

$\mathrm{L}_{\mathrm{R}}=\beta-\alpha ; \mathrm{f}_{2}(\mathrm{H}) \leq \mathrm{Q}_{\mathrm{L}}(\mathrm{H}) \leq \mathrm{f}_{1}(\mathrm{H})$

The use of the boundary curves $\mathrm{f} 1(\mathrm{H}) \& \mathrm{f} 2(\mathrm{H})$ allows the plotting within them of the Optimum, line, which acts as a visual check and allows qualitative interpretation of the results.

The optimization procedure described finds the particular straight line, within the permissible region $\mathrm{f} 2(\mathrm{H}) \leq \mathrm{QL}(\mathrm{H}) \leq$ $\mathrm{f} 1 \mathrm{H})$, which has a maximum horizontal projection on the head axis. That is, $\mathrm{Lr}$ is 118aximized. The optimization procedure described is applied for the two extreme cases, $\mathrm{P}$ $=0$ (Rectangular weir) and $\mathrm{P}=1$ (IVN as an orifice). The result obtained from the numerical optimization $(\mathrm{q}=0.6666667 \sqrt{ } 2 \mathrm{~g}(2 \mathrm{Wc}) \mathrm{h} 3 / 2)$ is the theoretical relationship for the rectangular weir $q=2 / 3 \sqrt{ } 2 \mathrm{~g}(2 \mathrm{Wc}) \mathrm{h} 3 / 2$.In the case of the IVN acting as an orifice the numerical result follows very closely the theoretical discharge head relationship. When tried upon a large variety of discharge-head curves the proposed optimization procedure gave consistently precise results. It can also be applied conveniently even when the discharge-head function is not explicitly known.

\section{EXPERIMENTS AT SSIT}

Experiments conducted at SSIT, Tumakuru confirm the constant value of coefficient of discharge with an average value obtained equal to 0.63 for $45^{\circ}$ value of $\theta$ with the same $d_{1}=0.12 \mathrm{~m}$. It was vital to ensure that similar results were obtained with change of conditions. The layout of the now demolished experimental setup can be obtained from Thesis of Ph.D students of IISc.The experimental setup in SSIT is shown in Figure and photo below:

At the weir grooves were made in the abutment of the channel. The weir seat was made perfectly horizontal using a highly sensitive spirit levels and the centerline of the channel was marked on it. The weir was slowly slid in to the groove and the weir was moved side-ways till the centerline of the weir coincided with that of the channel. The weir was inclined towards the outer edge of the groove and the gap between the plate and the inner edge was filled by petroleum wax on all the three sides of the weir both on the upstream and downstream of the weir section to avoid the leakage. Before starting the experiments, both the weir and the hook gauge were checked for their verticality with a plumb-bob and by a spirit level for their alignments. The flow over the 
notch was made to fall at the centre of the collecting tank by the channel cantilevering out from the support and adequate stilling arrangements were done by dumping graded boulders at the connecting point to ensure uniform rise in the piezometer. Water was pumped into a head tank of dimensions $(1.75 \mathrm{~m} * 1.20)$ Located at the head of the channel section by means of a centrifugal pump with a discharging capacity of $\left(\mathrm{m}^{3} / \mathrm{sec}\right)$. In case of large discharging weir, an additional pump with a discharging capacity of $\left(\mathrm{m}^{3} / \mathrm{sec}\right)$ was used.

The free discharge through the weir was collected in a measuring tank $(2.48 \mathrm{~m} * 1.8 \mathrm{~m})$. The time required to collect a fixed volume of water in the measuring tank was recorded with the help of a stop clock by attached to the level indicators located in a measuring tank of Perspex tube of $20 \mathrm{~mm}$ internal diameter. For each head over the weir crest, the actual discharge was calculated by taking an average of the time for six readings recorded by the stop clock (for each rise of $10 \mathrm{~cm}$ in the measuring tank).

The experiments were conducted on each weir both in increasing as well as decreasing order of the head. At least a time gap of 20 minutes was allowed between two sets of experiments to ensure a constant head. Further it was ensured that two head readings, taken with 10 minutes time gap did not exceed $0.1 \mathrm{~mm}$. The head readings were checked after every $0.10 \mathrm{~m}$ rise in the measuring tank.

Experiments were conducted on ten weirs with dimensions $W_{c}=6 \mathrm{~cm} ; d_{1}=9.22 ; \theta=29.2569^{\circ}$ In SI units, the headdischarge equation is:

$$
\begin{aligned}
& Q_{L}(\mathrm{H})=2.74626 d_{1}^{3 / 2} \tan \theta C_{d}\left(\mathrm{~h}+0.292 d_{1}\right) \mathrm{m}^{3} / \mathrm{sec} ; \\
& 0.900 d_{1} m \leq h \quad 7.470 d_{1}
\end{aligned}
$$

The eccentricities imparted were $0,4,8,12,16$ and $20 \mathrm{~cm}$, while the vertical displacement was 3.2, 6.2, 10.2 and 15.2, and $19.7 \mathrm{~cm}$ down to bed.

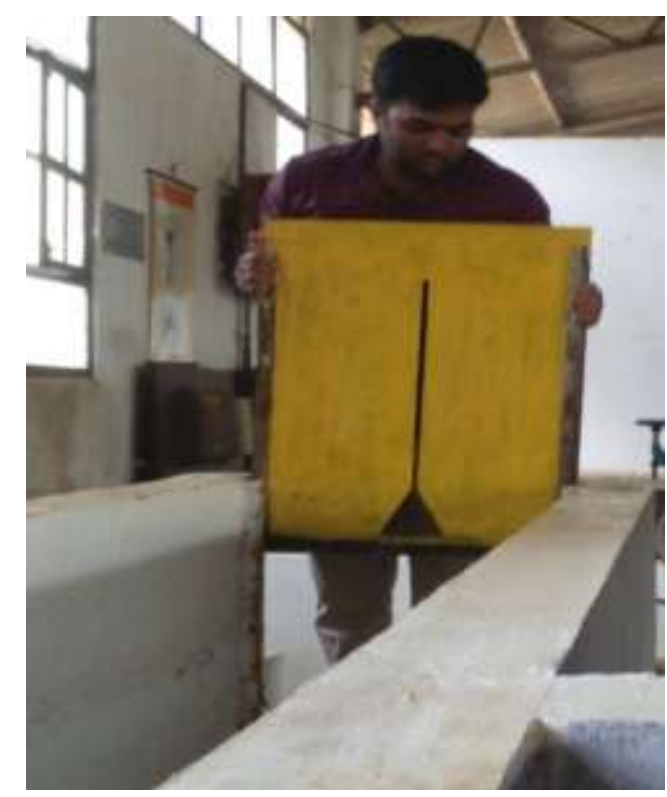

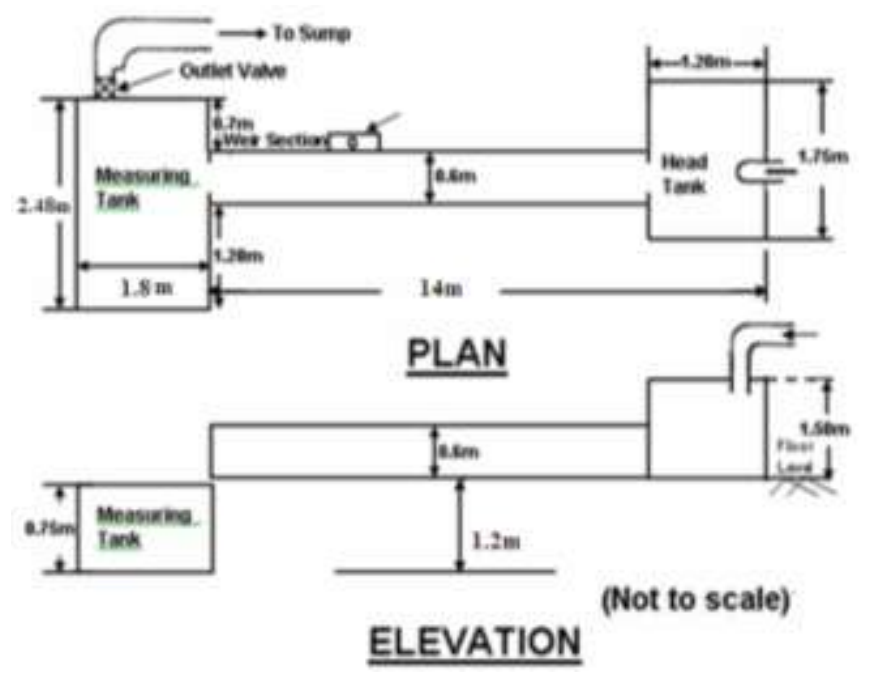

\section{ANALYSIS AND DISCUSSION OF RESULTS}

Table 1 Variation with $C_{d}$ with respect to eccentricity

\begin{tabular}{|l|l|l|l|}
\hline $\begin{array}{l}\text { Eccentricity } \\
(\mathrm{cms})\end{array}$ & Mean & $\begin{array}{l}\text { Standard } \\
\text { deviation }\end{array}$ & $\begin{array}{l}\text { Coefficient of } \\
\text { variation }\end{array}$ \\
\hline 0 & 0.6221 & 0.0164 & 2.63 \\
\hline 4 & 0.6438 & 0.0137 & 2.2127 \\
\hline 8 & 0.6141 & 0.022 & 3.582 \\
\hline 12 & 0.62 & 0.03604 & 5.833 \\
\hline 16 & 0.6201 & 0.0126 & 2.031 \\
\hline 20 & 0.655 & 0.01607 & 2.453 \\
\hline
\end{tabular}

Table 2 Variation with $C_{d}$ with respect to vertical shift from crest

\begin{tabular}{|l|l|l|l|}
\hline shift $(\mathrm{cms})$ & Mean & $\begin{array}{l}\text { Standard } \\
\text { deviation }\end{array}$ & $\begin{array}{l}\text { Coefficient of } \\
\text { variation }\end{array}$ \\
\hline 0 & 0.622 & 0.0164 & 2.63 \\
\hline 3.2 & 0.649 & 0.0286 & 4.406 \\
\hline 6.2 & 0.6591 & 0.01643 & 2.49 \\
\hline 10.2 & 0.6766 & 0.0228 & 3.369 \\
\hline 15.2 & 0.6668 & 0.02409 & 3.604 \\
\hline 19.7 & 0.6652 & 0.03555 & 5.34 \\
\hline
\end{tabular}

The linear fit COV for the case of horizontal shift COV : $\operatorname{COV}(\mathrm{e})=0.007 \mathrm{e}+3.032$ Therefore $\square(\mathrm{e}=20)=\operatorname{COV}(20)-$ $\operatorname{COV}(0)=0.14$

The Linear fit for COV for the case of vertical downward displacement

$\operatorname{COV}(\mathrm{y})=0.092 \mathrm{y}+3.032$
$\square(\mathrm{y}=20)=1.84$
Change in $\square=12.14$ or $1214 \%$

The reason why there is a dramatic increase in sensitivity to vertical displacement can be surmised by noting that the presence of turbulent rollers below the crest. The results indicate that the value of $C_{d}$ is hardly changed when the weir is moved to the sides. This is surprising because it was expected that the walls would influence the value of $C_{d}$ when the weir was moved towards extreme left or right. The distance of the corner of the weir from the channel wall was 
$4 \mathrm{~cm}$ in the extreme case. The boundary layer effect seizes at around $5 \mathrm{~cm}$. Next the effect of modification of curvature of stream lines by the side walls remains unaltered when the weir was shifted extreme left or right. This inference needs to be checked by flow visualization techniques which are beyond the scope of our work.

The reason why the vertical displacement led to such a dramatic increase in coefficient of variation (as compared to horizontal shift) can be explained easily by turbulent flow theory. It should be noted that the flow in open channels below the crest is highly turbulent. Turbulence imparts random fluctuations whose magnitude can be quiet large.

\section{CONCLUSION}

For all the weirs experimented on (10 weirs) the variation of $C_{d}$ was found to be \pm 1.5 errors, thus underlining the fact that the sharp crested weirs are indeed a very accurate instrument of flow measurement. The range of error is considered to be satisfactory if it is within 5 to $10 \%$ for other branches of hydrology. There is also no problem of locating the point of critical flow as in broad crested weirs. As was expected when the weir crest was made to coincide with the channel bottom the variation of $C_{d}$ with head was found to vary to a greater degree. The Cd was found to be extremely sensitive to vertical displacement as compared to horizontal displacement. The change in coefficient of variation was $1214 \%$

\section{ACKNOWLEDGEMENTS}

The Authors are indebted to VTU Belgavi for financial sanction under research grants scheme and SSIT Tumakuru for everything else to carry out the project. No words are enough to thank my guide Late Prof.K.Keshava Murthy my guide at IISc

\section{REFERENCES}

[1] C.Rangaraj Ph.D(1997)Thesis(IISc) Optimization \& design of proportional weirs

[2] Keshava Murthy, K. (1967), “A Generalized Mathematical Theory of Proportional Weirs", ph.D.Thesis, Indian Institute of Science, Bangalore.

[3] Keshava Murthy, K. (1969), "On the design of quadratic weirs", Journal of Franklin Institute, 287(2).pp. 159-174.

[4] Keshava Murthy, K. (1995), "Theory and Design of Proportional Weirs", D.Sc. thesis, Indian Institute of Science, Bangalore.

[5] Keshava Murthy, K. (1995A). "The Theory of Proportional Weirs", Journal of Indian Institute of Science, 75(3), pp.355-372.

[6] Keshava Murthy, K. and Giridhar.D.P (1989), "Inverted V-notch: A practical proportional weir", Journal of Irrigation and Drainage Division, American Society of Civil Engineers (ASCE), 115(6),pp. 10351050.

[7] Keshava Murthy, K. And Giridhar.D.P. (1990), "Improved Inverted V-notch or Chimney weir", Journal of Irrigation and Drainage Division, American Society of Civil Engineers(ASCE), 116(3),pp. 374-386.

[8] Keshava Murthy, K. And giridhar.d.p.(1991), "Geometrically simple linear weirs using circular quadrants: bell mouth weirs", International Association of Hydraulic Research(IAHR), Journal of Hydraulic Research, 29(4),pp.497-508.

[9] Keshava Murthy, K., Ramesh.H.S and Rangaraj.c. "Self-Basing Logarithmic Notch-Orifice", appearing in The International Journal for Engineering analysis and Design.

[10] Keshava Murthy, K., and Rangaraj.c. (1994), "Geometrically Simple Exponential Weir", Acta technical, Hungarian Academy of Sciences, Hungary, 106(3-),pp.223-238.

[11] Keshava Murthy, K.,and Rangaraj.c.(1997), "On the improvement of the chimney weir", Canadian Journal of Civil Engineering, National Research council, Canada, 24(4),pp.586-592 